Article

\title{
Synchrotron Radiation Research and Analysis of the Particulate Matter in Deep Ice Cores: An Overview of the Technical Challenges
}

\author{
Giannantonio Cibin ${ }^{1, *}$, Augusto Marcelli ${ }^{2,3}{ }^{(D)}$, Valter Maggi ${ }^{4,5}$ (D) , Giovanni Baccolo ${ }^{4,5}$ (D), \\ Dariush Hampai ${ }^{2}{ }^{\circ}$, Philip E. Robbins ${ }^{1}$, Andrea Lied1 ${ }^{2}$, Claudia Polese ${ }^{2}$, Alessandro D’Elia ${ }^{6}$, \\ Salvatore Macis ${ }^{2,7}$, Antonio Grilli ${ }^{2}$ and Agostino Raco ${ }^{2}$ \\ 1 Diamond Light Source, Harwell Science and Innovation Campus, Didcot OX110DE, UK \\ 2 Istituto Nazionale di Fisica Nucleare, Laboratori Nazionali di Frascati, via Enrico Fermi 40, Frascati, \\ I-00044 Roma, Italy \\ 3 Rome International Centre for Material Science Superstripes, RICMASS, via dei Sabelli 119A, \\ I-00185 Rome, Italy \\ 4 Dipartimento di Scienze dell'Ambiente e della Terra, Università degli Studi di Milano Bicocca, \\ Piazza della Scienza, I-20126 Milano, Italy \\ 5 Istituto Nazionale di Fisica Nucleare, Sezione di Milano-Bicocca, Piazza della Scienza, 2, I-20126 Milano, Italy \\ 6 Department of Physics, University of Trieste, Via A. Valerio 2, I-34127 Trieste, Italy \\ 7 Department of Physics, Università Sapienza, Piazzale Aldo Moro 5, I-00185 Rome, Italy \\ * Correspondence: giannantonio.cibin@diamond.ac.uk; Tel.: +44-1235-778645
}

Received: 26 April 2019; Accepted: 24 June 2019; Published: 27 June 2019

check for updates

\begin{abstract}
Airborne dust extracted from deep ice core perforations can provide chemical and mineralogical insight into the history of the climate and atmospheric conditions, with unrivalled temporal resolution, time span and richness of information. The availability of material for research and the natural complexity of the particulate, however, pose significant challenges to analytical methods. We present the developments undertaken to optimize the experimental techniques, materials and protocols for synchrotron radiation-based analysis, in particular for the acquisition of combined Synchrotron Radiation X-Ray Fluorescence and X-ray Absorption Spectroscopy data.
\end{abstract}

Keywords: synchrotron radiation; ice core, atmospheric mineral dust; X-ray absorption spectroscopy

\section{Introduction}

The same phenomena that make deep ice cores precious archives of environmental information are at the source of the technical challenges that studies of chemical, isotopic, mineralogical composition face. This is particularly true where ice cores are used for the acquisition of data aiming at accurate reconstructions of the temporal evolution of atmospheric conditions. Glacier location, atmospheric circulation patterns, ice accumulation rates and the local meteorological conditions determine whether ice preserves, in a time-ordered sequence, samples of the deposited snow. The combined effect of accumulation rate, glacier dynamic, local climatic conditions and geothermal heat flux define the maximum timespan of the preserved ice. The low deposition rates and overall low temperature conditions in Antarctica guarantee, therefore, maximal time span records, but this impacts on the achievable temporal resolution; at the same time, the long distance from sources of airborne particulates and the snow capture mechanisms limit the overall concentration of atmospheric components that precipitate and get stored in ice. Snow deposition rates in Antarctica can be as low as $50 \mathrm{~mm}$ equivalent water per year, and this allows deep ice cores to cover past climates back to several hundred ka [1]. Because of the coverage thickness, the accumulation usually leads to unperturbed storage which are less 
affected by lateral ice movements (when compared e.g., to alpine environments). Also, the provenance of solid material found in ice is atmospheric or meteoric, so Antarctic ice collects non-local information.

Climate and atmospheric information from deep ice cores is first obtained from accurate analysis of ice itself, in particular through the measurement of deuterium and oxygen stable isotope composition, ionic concentrations and gas analysis (e.g., Petit [1]). These parameters provided a direct measurement of temperature across the last few climatic cycles.

Solid (insoluble) particulate represents a significant fraction among the ice core components, as its compositional analysis can reveal temporal variations of the activity of dust sources and of their environmental conditions. Those have consequences on global bio geochemical cycles, and influence mineral alteration under different climatic conditions [2].

Dust content and grain size measurements performed using liquid counter methods $[1,3]$ have indicated that the dust deposition rate also has a close correlation with climate. The rate reflects the evolution of dust sources and of the atmospheric circulation patterns. Comparative analyses with samples from potential source areas, using TIMS (Thermal Ionization Mass Spectrometer) isotopic analysis on the ratios ${ }^{87} \mathrm{Sr} /{ }^{86} \mathrm{Sr}$ and ${ }^{143} \mathrm{Nd} /{ }^{144} \mathrm{Nd}$, has indicated a South American provenance of dust deposited in inner East Antarctica [4] in cold periods.

Beyond elemental composition information, obtaining a mineralogical analysis of the particulate would naturally complement these results with the potential of giving much richer insight into both the source and the transport phenomena across the last climatic periods [5-8]

Technical challenges clearly come from the small amounts of solid material available for analysis. The particulate concentration in ice $[9,10]$ in deep ice cores from Antarctic drilling locations, for example, oscillates, depending on the climatic period samples, with dramatic variations from $500 \mathrm{ng} / \mathrm{g}$ down to $20 \mathrm{ng} / \mathrm{g}$ during glacial and interglacial periods, respectively. These concentrations of particulate in ice, however, are well below the detection limits of modern mineralogical analytical techniques. Extracting significant amounts of the insoluble fraction for chemical and mineralogical analysis therefore requires careful handling and clean protocols; at the same time, it is necessary to minimize the ice core sections used for extraction, considering both the sample rarity (deep ice core drilling present significant challenges involving international collaborations [4]) and the need to preserve the time resolution, directly linked to the ice core section depth profile being sampled. The amounts of particulate extracted will be necessarily minimal. We will introduce here the development in instrumentation and protocols undertaken by our group to optimize the collection of synchrotron radiation X-ray Fluorescence (XRF), X-ray Absorption Spectroscopy (XAS) and X-ray Powder Diffraction (XRD) data on such samples.

\section{Methods}

\subsection{Elemental Composition Analysis}

Elemental composition analysis of particulate from deep ice cores has been undertaken before, using Particle-Induced X-ray emission (PIXE) [11,12]. Similar to other X-ray emission detection methods, PIXE analysis provides concentration information for most elements (in practice all elements heavier than $\mathrm{Na}$ ), has a high sensitivity so is suitable for detecting components in low concentration and is non-destructive for mineral samples. However, PIXE information is limited to elemental analysis, and the exposure to high-energy proton beams has direct effects on the stability of the supports. This possibly precludes re-using the samples in further analyses. As an alternative to proton-induced emission, X-ray based methods can overcome these limitations. Lab-based instrumentation makes it possible to detect trace element concentrations in particular, with the Total Reflection X-Ray Fluorescence configuration, exploiting the advantage that such a configuration minimizes the contribution from sample supports and enhances the signal coming from the materials deposited on flat surfaces. The growing importance of XRF and related techniques is demonstrated by the continuous development and size of its community; advances on materials, technology and procedures are regularly reported in contributions published on J. Anal. At. Spectrom. [13]. The use of synchrotron-based instrumentation 
clearly provides substantial advantages due to the availability of intense, highly collimated and, most importantly, tunable monochromatic beams, at the expense of beam time availability and limited access due to intense competition for access to synchrotron beamlines. Finally, X-ray based analyses have the important characteristics that they don't alter the sample (radiation damage in mineral systems is not as critical as for other sample matrices).Multiple techniques, make it possible to get structural rather than elemental information, and they can be applied on the same sample, provided that sample preparation is compatible with different experimental needs. The increasing importance of synchrotron radiation is evidenced by the increasing list of instruments dedicated to XRF and related methods. A comprehensive list of XRF beamlines is reported in the recent paper from Karydas et al. [14]. It is worth mentioning, however, that recent beamline development efforts are mostly aimed at reducing the beam sizes, due to the scientific potential of microscopic methods e.g., in the environmental and life sciences. Those experiments are particularly demanding in terms of beamtime allocation times, and do not guarantee the efficient use of beam times when average XRF and XAS information is needed, as in our case.

\subsection{Sample Preparation and Handling}

Dust extraction from ice core samples was undertaken in a clean room environment at the EuroCold Lab facility, University Milano-Bicocca. After the removal with three successive baths in ultra-pure water of the contaminated core surface, ice samples were melted at room temperature in a laminar flow bench and dust was extracted by filtration [5]. All materials used during this procedure are rinsed with ultrapure water and undergo a deep cleaning with hyper-pure nitric acid. The choice of materials and the cleaning procedures have been developed over time, and a detailed analysis of the filter materials using Neutron Activation Analysis has been recently reported in the papers of Baccolo et al. [12]. For our synchrotron experiments dust was deposited on polycarbonate filters with $0.45 \mu \mathrm{m}$ pore size. Filters were mounted on clean polytetrafluoroethylene holders. The effectiveness of the cleaning protocol was verified on the beamline by comparing XRF measurements on filters as-bought, after the cleaning procedure and filtering the ultrapure water used for container rinsing.

Considering that beamline measurement spaces are not usually prepared to operate in clean-room conditions, we developed a protocol to minimize sample contamination during transport and handling after filtration and during the preparation carried out in the EuroCold laboratory [15]. The samples were transported in triple containment, in heat-sealed clean polyethylene bags. Outer and intermediate containments were removed in Diamond clean room and sealed samples transferred to the beamline in clean sealed containers. To allow a clean introduction of samples into the experimental chamber, the latter was extended with an ambient pressure glove box (built in-house), nitrogen-filled, with internal access to the experimental chamber sample load door. The last containment layer was removed just before insertion of the PTFE filter support to the chamber holder with the help of clean tweezers. After measurement, the samples were again sealed in the original envelopes before being returned to the clean chamber for long-term storage. While this handling sequence is probably not critical for experiments programmed in individual batches, it allows repeated measurements to be undertaken in different experimental runs. Considering the intrinsic variability (including seasonal oscillations) of natural dust composition deposited in ice cores, running acquisition campaigns in separate experimental visits has allowed us to refine the sample selection on the basis of previous measurements, and to assess the nature of outliers in the temporal sequence being analyzed. Maintaining clean sample conditions after measurement has therefore allowed cross-checking of the experimental setups to occur, ensuring compatibility of the results across different experiments.

For each experimental run, blank filters were prepared using the same methods as the dust samples, and SR-XRF data were collected to validate the experimental run and ensure that during transport or storage, no significant contamination occurred. Also, a calibration batch of reference standards prepared with comparable amounts of NIST reference soil (NIST 2709a, San Juaquin soil [16]) was regularly measured. 


\subsection{Experimental}

For this work, we had access to two facilities: the Stanford Synchrotron Radiation Lightsource (beamlines 6-2 and 7-2) and Diamond Light Source (beamline B18). In both cases, the beamlines were equipped with double-crystal monochromators, equipped with Si(111) crystals. At SSRL the beam, the liquid-nitrogen cooled monochromators were focused with mirrors in the vertical direction to give a line beam profile of the sample for grazing-incidence data collection, with a beam vertical size of approximately $200 \mu \mathrm{m}$. The experimental chamber for total reflection fluorescence and GI-XAS in vertical deflection geometry was developed under the CRYOALP [12] program. Data were acquired using a Silicon Drift detector from KETEK GmbH (Munchen, UK) with Canberra (now Mirion technologies, Meriden, CT, USA) electronics. On B18, the beam was focused horizontally with a FWHM of $200 \mu \mathrm{m}$ for Total reflection X-Ray Fluorescence and Grazing Incidence X-ray Absorption Spectroscopy acquisitions in the horizontal plane, and defocused to give a $1 \times 1 \mathrm{~mm}^{2}$ beam footprint on the sample. Membrane samples were aligned for conventional $45^{\circ}$ incidence measurements. Data were acquired with a Vortex 4-elements silicon drift detector from SII (Hitachi High Technologies Science America, Nothridge, CA, USA), and Xmap (XIA LLC, Hayward, CA, USA) and XSPRESS3 (Quantum Detectors, Didcot, UK) electronics.

\section{Results and Discussion}

\subsection{X-Ray Fluorescence and X-Ray Absorption Spectroscopy in Grazing Incidence Geometry}

When the critical information to be extracted from a sample is coming from a surface, as in the case of particulate deposited on a solid flat substrate, orienting the latter so the incoming beam is in grazing incidence geometry substantially reduces the interaction with the support thanks to X-ray total external reflection. In particular, the substrate does not generate diffuse scattering if its surface roughness is low. The reflection critical angle, which allows the total reflection regime to be established, depends on the substrate composition and X-ray beam energy. This setup takes advantage of a two-fold increase in the amplitude signal obtainable from the creation of a stationary wave on the reflecting surface. Finally, in this geometry, the beam footprint on the support gets significantly increased, allowing for the detection of large surface sections.

We collected our initial set of data on Antarctic particulate samples at SSRL (beamlines 6-2 and 7-2) using a dedicated experimental chamber [17], and at Diamond Light Source (beamline B18). In Figure 1 we compare XRF results from samples deposited on filters and analyzed through normal incidence geometry with the ones deposited on Si wafers and measured in total reflection geometry. The single measurement efficiency and data quality obtainable in total reflection mode are significantly improved, as expected. The total reflection X-ray Fluorescence (TXRF) geometry allows us to probe a large surface area, as the beam footprint on the sample is extended thanks to the $1 / \sin (\theta)(\theta$ being the grazing incidence angle) geometrical factor; however, several considerations come into play. First, this setup requires dedicated experimental systems, and total reflection setups are not commonly available at XAS beamlines. This means that for long term programs, specialised chambers must be developed and integrated with different synchrotron radiation facilities, and logistics aspects thus have to be considered.

Other limitations of the TXRF method are: the need of focusing the beam down to a few hundred microns; time consumption for the sample alignment, and the importance of keeping the beam position extremely stabile during the measurements, in particular during XAS scans. In addition, the grazing incidence angle must be adjusted with high accuracy, as the fluorescence line intensity of elements present in the support is critically dependent on the angle. This is clearly essential in our case, as the XRF analysis must include an accurate quantification of Silicon, which is present in the common clean materials used as supports for TXRF analysis (commonly used are quartz and Si wafers). Alternative materials to avoid interference with Si and most of the elements of interest for natural particulate analysis are in practice not available; in particular, we found that plastic-based supports with had 
contaminations, inferior surface quality and the total reflection angles were significantly lower than Si. Significantly heavier elements present lower energy absorption edges ( $\mathrm{L}$ and $\mathrm{M}$ ) that always fall into the region of interest for major element analysis in natural systems. The deposition of particulate on substrates requires additional manipulation steps, such as removal from filters with ultrapure water and re-deposition on substrates, with additional risks of contamination. This deposition method causes a visible "coffee stain" pattern on the substrate, which could lead to a fractionation of different components leading to different results, depending on the part of the sample illuminated by the beam [17].

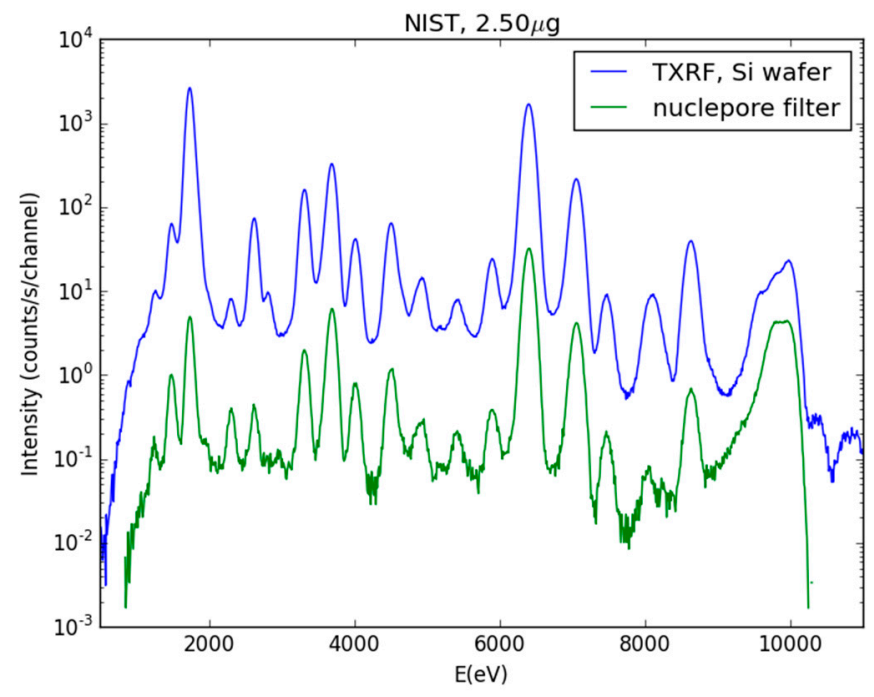

Figure 1. Comparison between TXRF and normal incidence XRF acquired on reference NIST soil samples, with same amounts deposited. Apart from the overall higher intensity, the TXRF measurement displays a significantly different Si signal intensity due to the substrate contribution, and at the same time a lower elastic scattering contribution to the spectrum. The remaining signal from other elements is consistent across the two measurements, indicating the background contribution from filter contamination is negligible.

\subsection{Normal Incidence Geometry}

On the opposite side, the use of polymer membrane filters presents issues coming from the nature of the material and the experimental arrangements. The incoming beam probes the whole support thickness, so composition contamination of the filters will be more important than in total reflection geometry (Figure 2 reports a comparison between XRF acquired on two blank filters-PMMA and metacrylate-and a standard soil sample prepared depositing the smallest amount used in our research). Minimizing the filter thickness is important as well. This does not only help with the direct contamination signal, but helps reducing the overall diffuse beam scattering (a limiting factor for fluorescence detectors for dilute components analysis). It is expected that most of the fluorescence signal also from the low $\mathrm{Z}$ components should come from the front filter surface, so corrections for the low energy efficiency are considered minor.

Regarding the sample preparation, filtering does not pose the same problems regarding potential separation between components due to coffee stain. However, if the sample is deposited approximately on the same surface (as with the use of common lab filtering systems, with a deposition diameter of $13 \mathrm{~mm}$ ), the sample density "seen" by the beam will be significantly lower, as discussed.

At the same time, filtration on samples characterized by small particle numbers does not guarantee a homogeneous deposition. In Figure 3, we present the composition profile acquired on a filter prepared with this method. It is clear that significant variations across the sample diameter are present, i.e., up to $50 \%$ for the $\mathrm{Ca} / \mathrm{Fe}$ ratio. 


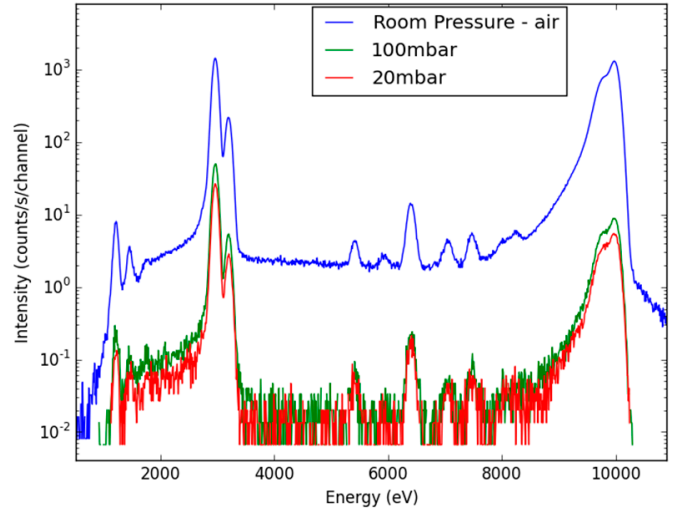

(a)

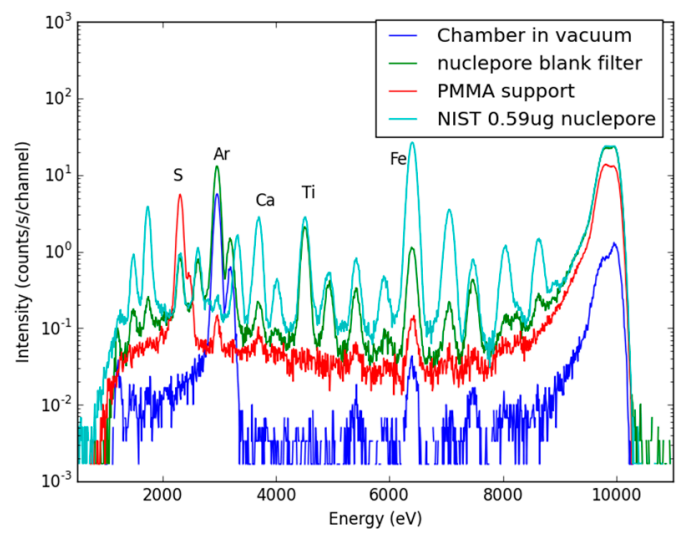

(b)

Figure 2. (a) Variation of background signal in vacuum and atmospheric pressure conditions from the experimental chamber. The atmospheric pressure measurement shows, beyond the strong contribution of Ar and direct scattering, a strong enhancement of signals coming from fluorescence from steel components present in the experimental chamber materials, excited by the diffuse scattering. Visible lines are $\mathrm{Ar} \mathrm{K} \alpha$ and $\mathrm{K} \beta$ emission lines (main and escape peaks at low energy), $\mathrm{Cr}$, Fe and $\mathrm{Ni}$. (b) Comparison between the experimental chamber background, spectra from one of many the polymer supports considered (PMMA, polymethyl metacrilate) showing traces of sulfur contamination, a Nuclepore polycarbonate blank filter before acid cleaning and a dilute NIST 2709a sample, consisting in $0.59 \mu \mathrm{g}$ mineral particles deposited on Nuclepore filter.

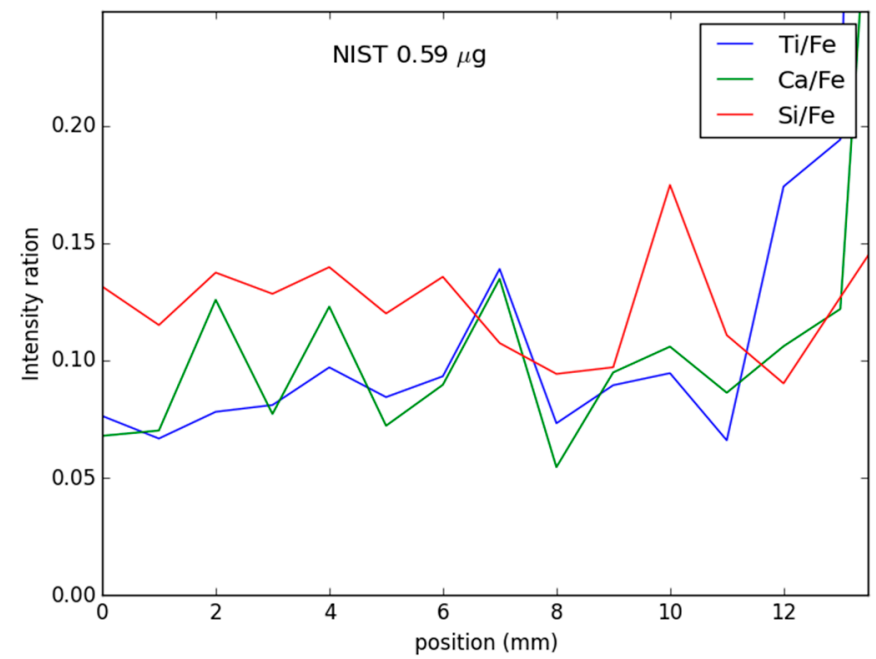

Figure 3. Intensity ratios between different elemental components extracted from XRF spectra taken at different vertical positions on a low concentration $\left(0.59 \mu \mathrm{g} / \mathrm{cm}^{2}\right)$ sample, deposited on nuclepore filtersover a $13 \mathrm{~mm}$ diameter area. The total beam footprint on the sample was adjusted to match the experimental step $\left(1 \times 1 \mathrm{~mm}^{2}\right)$. The variability in the concentration ratios highlights that potential composition variation artifacts could be introduced if the sample deposition happens on large surfaces.

This can be moderated with a reduction of the deposition area, and a change of the deposition method to concentrate the sample in the smallest possible area of the filter surface. An additional step in this direction has been undertaken by considering automated deposition systems [18], which proved to be efficient in minimizing the amount of ice necessary to reach a desired sample concentration on small areas (few $\mathrm{mm}^{2}$ ) that are directly comparable with the beam sizes attainable on modern focused beamlines. This deposition could dramatically improve the homogeneity of the deposited material and, at the same time, reduce both the deposition time and sample consumption, and increase the local concentration of the sample on the filter. For XRF analysis, a limiting factor for reducing 
the detection limit is the presence of an experimental background signal. Several aspects affecting the signal quality were taken into consideration. The general source for the background was found to be diffuse scattering, causing secondary emission from the experimental chamber wall materials, while contamination coming from the filters' support was dealt with by using filter supports and sample holders all manufactured in PTFE. Reduction of the scattering intensity and minimization of the background signal has been obtained by:

(a) Reducing the low level, diffuse, out-of-focus contribution from the beamline optics (caused by mirror coatings roughness and vacuum windows) using beam stripping slits placed just before the ionization chamber monitoring the incoming beam intensity;

(b) Reducing further contributions from small angle scattering by the ionization chamber windows (Kapton, thickness: $50 \mu \mathrm{m}$ ), by modifying the position of the ion chamber, increasing its distance to the entrance of the experimental chamber and adding a plastic collimator at the entrance of the chamber. The collimator, while still allowing for scanning the experimental chamber to map the sample position, ensured any diffuse beam to propagate through the sample position to the end aperture of the vacuum chamber (Figure 4);

(c) Reducing diffuse scattering from the residual gas in the vacuum chamber, improving the pumping system (experiments were always run under turbo-molecular pumping, ensuring a measurement pressure lower than 1 mbar);

(d) Filtering the fluorescence from the chamber walls by covering the internal walls with a 2 mm-thick polycarbonate lining and ensuring the beam exit path is free from metal sections close to the diffuse scattering from the sample support;

(e) Finally, minimizing the collection field of view by adding a clean PTFE collimator placed in front of the 4-elements SDD detector.

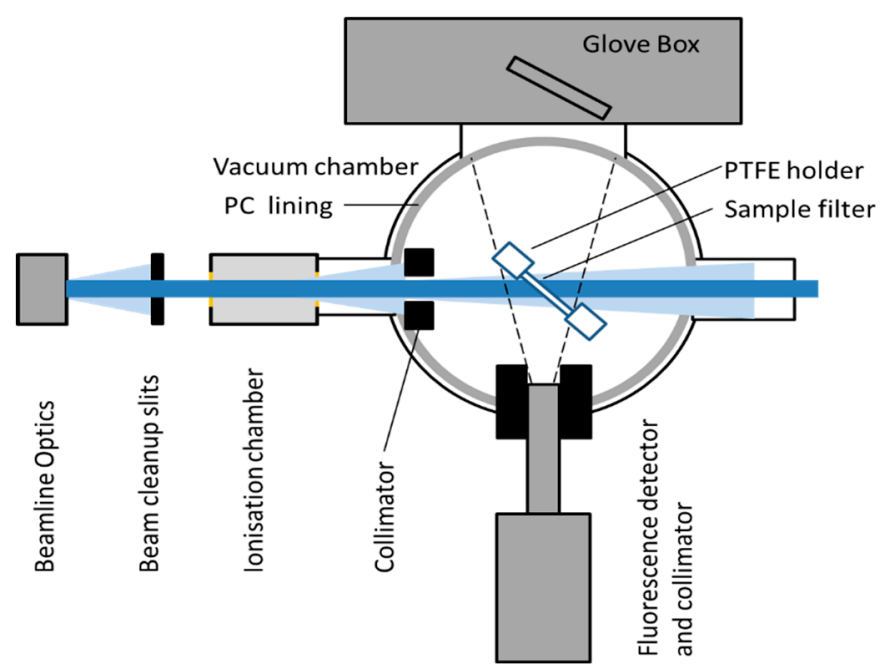

Figure 4. Layout of the experimental setup. In black are indicated the beam collimation components (cleanup slits, experimental chamber and fluorescence detector collimator), reducing the spatial distribution of beam diffused by optical elements and windows (in light blue). In light grey, the vacuum chamber lining in polycarbonate used to minimize contributions of $\mathrm{X}$-ray fluorescence from the experimental chamber, excited from beam scattered from the filter and sample support.

\subsection{X-Ray Absorption Spectroscopy}

The optimization of the experimental setup for XRF measurements allowed us to collect, in an efficient way, a full set of X-ray Absorption Spectroscopy data at the edge of elements of interest to obtain the information on oxidation states and element-specific coordination that XAS provides to support, in particular, through direct comparison with relevant standards, the identification of the 
mineral composition of our samples [11]. In particular, we were able to collect data at the Fe, $\mathrm{Ti}$ and $\mathrm{Ca}$ $\mathrm{K}$ edges on a significant set of samples, across the last climatic transition, during a long-term proposal on B18 (Figure 5). These elements are important in the case of ice core particulate in Antarctica, as information on Fe speciation can lead to an identification of the dust sources. The relationship between $\mathrm{Ti}$ and Fe chemistry brings information on local vs. long range transport in Antarctica, and Ca can be of continental or marine origin. The samples' concentration was optimized to give a significant total photon count rate on the fluorescence detector, which was limited to approximately $200 \mathrm{kcps} / \mathrm{element}$ over all the project run to ensure all samples data were acquired in the same experimental conditions (in particular, the same maximum detector dead time fraction which is dependent on the total count rate). The total signal from polycarbonate filters at the energies corresponding to the Fe $\mathrm{K}$ edge absorption edge measurements (up to $7.8 \mathrm{keV}$ ) was found to always be in the ideal detector range, with samples containing total amounts of particulate in the range of $\sim 3-17 \mu g$, distributed over an approximate surface of $0.25 \mathrm{~cm}^{2}$. It is worth noting that this indicates (at least for the Fe $\mathrm{K}$ edge) that no significant advantages would be attainable from the use of more intense sources, such as wiggler/undulator based beamlines, as the experiment duration would be detector-limited. An optimal acquisition time efficiency was obtained using a continuous energy scanning mechanism-already developed on B18 - so minimal dead times during acquisitions were present. For the Fe K edge, a total acquisition time of up to $\sim 15 \mathrm{~min}$ was sufficient for extended XANES spectra (data were collected usually up to $7500 \mathrm{eV}$ to ensure accurate normalization, and up to $7800 \mathrm{eV}$ for a reduced number of concentrated samples to allow for EXAFS evaluation).

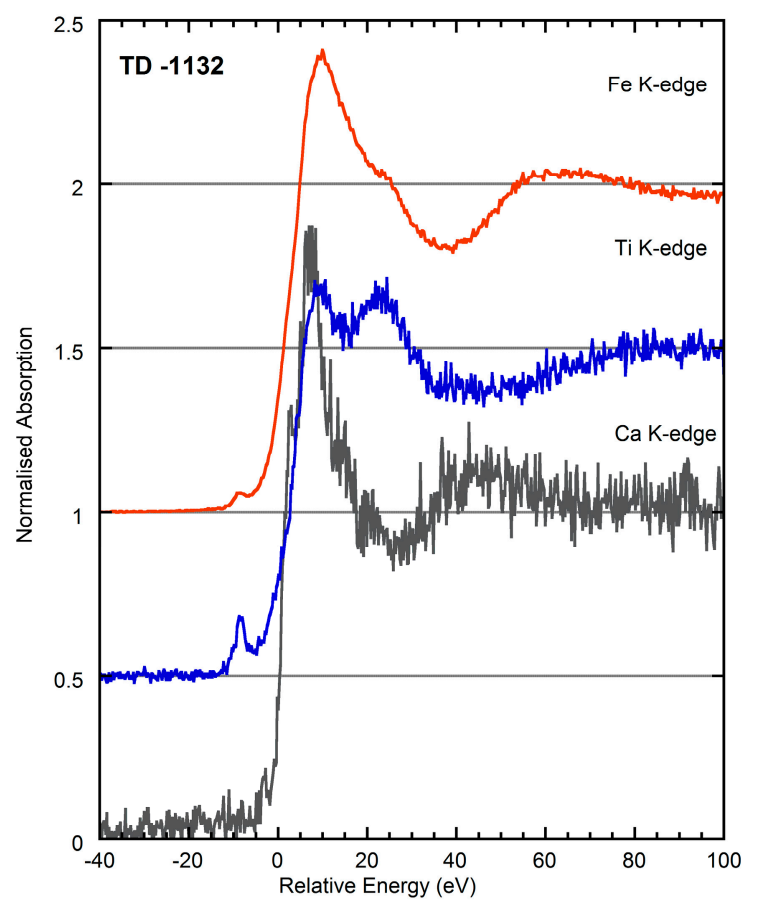

Figure 5. X-ray Absorption spectra collected at the Fe, Ti and Ca K-edge on a representative sample $\left(1.2 \mu \mathrm{g} / \mathrm{cm}^{2}\right.$ of Antarctic dust on a polycarbonate membrane) from Talos Dome. The energy axis is relative to respective absorption edges. The total acquisition time for Fe K-edge was $12 \mathrm{~min}$, while for $\mathrm{Ti}$ and $\mathrm{Ca}$ we collected data for 30 and $45 \mathrm{~min}$, respectively.

Using continuous scans [19], we could collect several measurement repetitions over short time periods (approximately $3 \mathrm{~min} / \mathrm{scan}$ ), which allowed us to monitor potential radiation-induced effects such as photoreduction/oxidation, and to adjust the total acquisition time per sample to obtain a homogeneous data quality across the whole sample set. With the SDD detector placed at $\sim 50 \mathrm{~mm}$ from the sample surface, it was possible during the same experimental session to acquire XANES data 
from lower energy edges (Ca and Ti), with sufficient quality to allow for a good recognition of the coordination environment of those two elements. It is important to note that the signal drops rapidly at low energies, because of the lower fluorescence yield, so acquisition times were proportionally longer (we limited our collection time to $1 \mathrm{~h} /$ sample).

This setup allowed us to collect a full set of XRF and XAS data on the whole timespan covered by the Talos Dome perforation, located on the East Antarctic Ice Sheet [20]. This is a set of over 200 XRF and XAS individual acquisitions, which includes a significant fraction of data taken on background, blanks, and repetition measurements made necessary by the need to ensure that reproducible experimental conditions are met for all the experiments, split across several visits over a long-term project at Diamond Light Source. The first experiments were dedicated exclusively to the development and optimization of the acquisition and sample preparation, and determined, for example, the strategy of acquiring only data from filter samples, excluding the route of grazing-incidence measurements given the experimental uncertainties evidenced in the first runs.

\section{Conclusions}

The analysis of the insoluble fraction deposited in deep ice cores requires the development of techniques and protocols which are specific to this field. The small amounts of material available for analysis represent only one of the aspects to be taken into consideration when planning experimental campaigns, in particular if they rely on instrumentation available at large scale facilities. Instrumentation development in close collaboration with the facility staff substantially reduces the detection limits of the techniques, but careful sample preparation must be taken into consideration from the beginning, and must be an integral part of the experimental plans. The facts that access to beamtime is restricted and experimental campaigns could be divided in successive experimental visits, which are necessary to collect enough experimental points in the timescale covered by deep ice core to give statistically significant results, challenge the reproducibility of the instrumentation and of the preparation procedure, and require careful cross-checking and validation. We achieved a robust analysis set of consistent data on deep ice cores by incrementally developing these methods, thanks to a long term and close collaboration of all the parties involved, both from the scientific and technical sides, and by dedicating significant part of the beamtime to the analysis of instrument performance via long-term experimental campaigns.

Author Contributions: conceptualization, A.M., G.C., V.M.; methodology, A.M., D.H., G.C., G.B., P.E.R., A.R., A.G., S.M.; investigation, A.M., D.H., G.C., G.B., A.L., C.P., S.M.; software, G.C., D.H., G.B.; resources, A.M., V.M., G.B., G.C.; formal analysis, G.C., G.B., D.H., C.P., A.L.; data curation, G.C., D.H., C.P., G.B., A.L.; writing-original draft preparation, G.C.; writing-review and editing, all authors; supervision, project administration, funding acquisition, A.M., V.M.

Funding: Part of the results presented were acquired at the Stanford Synchrotron Radiation Lightsource, SLAC National Accelerator Laboratory, supported by the U.S. Department of Energy, Office of Science, Office of Basic Energy Sciences under Contract No. DE-AC02-76SF00515. Part of the work is done using specific funding by Dipartimento Affari Regionali e Autonomie (DARA) of the Italian Presidenza del Consiglio dei Ministri.

Acknowledgments: We are grateful for the support of SSRL staff, in particular Piero Pianetta and Matthew Latimer for helping with the beamline setup at 6-2 and 7-2. The authors wish to acknowledge Diamond Light Source for provision of beamtime within proposals sp7314, sp8372 and sp9050. We sincerely acknowledge Annibale Mottana for having triggered these researches and for his continuous support.

Conflicts of Interest: The authors declare no conflict of interest. 


\section{References}

1. Petit, J.R.; Jouzel, J.; Raynaud, D.; Barkov, N.I.; Barnola, J.M.; Basile, I.; Bender, M.; Chappellaz, J.; Davis, M.; Delaygue, G. Climate and Atmospheric History of the Past 420,000 Years from the Vostok Ice Core, Antarctica. Nature 1999, 399, 429-436. [CrossRef]

2. Maher, B.; Prospero, J.M.; Mackie, D.; Gaiero, D.; Hesse, P.P.; Balkanski, Y. Global connections between aeolian dust, climate and ocean biogeochemistry at the present day and at the last glacial maximum. Earth Sci. Rev. 2010, 99, 61-97. [CrossRef]

3. Delmonte, B.; Andersson, P.S.; Hansson, M.; Schoeberg, H.; Petit, J.; Basile-Doelsch, I.; Maggi, V. Aeolian dust in East Antarctica (EPICA-Dome C and Vostok): Provenance during glacial ages over the last $800 \mathrm{kyr}$. Geophys. Res. Lett. 2008, 35, L07703. [CrossRef]

4. EPICA community members. Eight glacial cycles from an Antarctic ice core. Nature 2004, 429, $623-628$. [CrossRef] [PubMed]

5. Sala, M.; Sala, M.; Delmonte, B.; Frezzotti, M.; Proposito, M.; Scarchilli, C.; Maggi, V.; Artioli, G.; Daoiaggi, M.; Marino, F.; et al. Evidence of calcium carbonates in coastal (Talos Dome and Ross Sea area) East Antarctica snow and firn: Environmental and climatic implications. Earth Planet. Sci. Lett. 2008, 271, 43-52. [CrossRef]

6. Briat, M.; Royer, A.; Petit, J.R.; Lorius, C. Late glacial input of eolian continental dust in the Dome C ice core: Additional evidence from individual microparticle analysis. Ann. Glaciol. 1982, 3, 27-30. [CrossRef]

7. Gaudichet, A.; Angelis, M.D.; Lefevre, R.; Petit, J.R.; Korotkevitch, Y.S.; Petrov, V.N. Mineralogy of insoluble particles in the Vostok Antarctic ice core over the last climatic cycle (150 kyr). Geophys. Res. Lett. 1988, 15, 1471-1474. [CrossRef]

8. Dapiaggi, M.; Sala, M.; Artioli, G.; Fransen, M.J. Evaluation of the phase detection limit on filter-deposited dust particles from Antarctic ice cores. Zeit. Kristallog. Kristallogr. Suppl. 2007, 26, 73-78. [CrossRef]

9. Lambert, F.; Delmonte, B.; Petit, J.R.; Bigler, M.; Kaufmann, P.R.; Hutterli, M.A.; Stocker, T.F.; Ruth, U.; Steffensen, J.P.; Maggi, V. Dust-climate couplings over the past 800,000 years from the EPICA Dome C ice core. Nature 2008, 452, 616-619. [CrossRef] [PubMed]

10. Wegner, A.; Fischer, H.; Delmonte, B.; Petit, J.R.; Erhardt, T.; Ruth, U.; Svensson, A.; Vinther, B.; Miller, H. The role of seasonality of mineral dust concentration and size on glacial/interglacial dust changes in the EPICA Dronning Maud Land ice core. J. Geophys. Res. Atmos. 2015, 120, 9916-9931. [CrossRef]

11. Marino, F.; Maggi, V.; Delmonte, B.; Ghermandi, G.; Petit, J. Elemental composition (Si, Fe, Ti) of atmospheric dust over the last $220 \mathrm{kyr}$ from the EPICA ice core (Dome C, Antarctica). Ann. Glaciol. 2004, 39, 110-118. [CrossRef]

12. Baccolo, G.; Maffezzoli, N.; Clemenza, M.; Delmonte, B.; Prata, M.; Salvini, A.; Maggi, V.; Previtali, E. Low background neutron activation analysis: A powerful tool for atmopsheric mineral dust analyisis in ice cores. J. Radioanal. Nucl. Chem. 2015, 306, 589-597. [CrossRef]

13. Vanhoof, C.; Bacon, J.R.; Ellis, A.T.; Vincze, L.; Wobrauschek, P. 2018 atomic spectrometry update-A review of advances in X-ray fluorescence spectrometry and its special applications. J. Anal. At. Spectrom. 2018, 33, 1413-1431. [CrossRef]

14. Karydas, A.G.; Czyzycki, M.; Leani, J.J.; Migliori, A.; Osan, J.; Bogovac, M.; Wrobel, P.; Vakula, N.; Padilla-Alvarez, R.; Menk, R.H.; et al. An IAEA multi-technique X-ray spectrometry endstation at Elettra Sincrotrone Trieste: benchmarking results and interdisciplinary applications. J. Synchrotron Radiat. 2018, 25, 189-203. [CrossRef] [PubMed]

15. EuroCold Lab. Available online: http://www.eurocold.unimib.it/ (accessed on 1 June 2019).

16. Mackey, E.A.; Christopher, S.J.; Lindstrom, R.M.; Long, S.E.; Marlow, A.F.; Murphy, K.E.; Paul, R.L.; Popelka-Filcoff, R.S.; Rabb, S.A.; Sieber, J.R.; et al. Certification of Three NIST Renewal Soil Standard Reference Materials for Element Content: SRM 2709a San Joaquin Soil, SRM 2710a Montana Soil I, and SRM 2711a Montana Soil II; NIST Special Publication 260-172; National Institute of Standards and Technology: Gaithersburg, MD, USA, 2010; p. 39. [CrossRef]

17. Cibin, G.; Marcelli, A.; Maggi, V.; Sala, M.; Marino, F.; Delmonte, B.; Albani, S.; Pignotti, S. First combined total reflection $\mathrm{X}$-ray fluorescence and grazing incidence $\mathrm{X}$-ray absorption spectroscopy characterization of aeolian dust archived in Antarctica and Alpine deep ice cores. Spectrochim. Acta Part B At. Spectrosc. 2008, 63, 1503-1510. [CrossRef] 
18. Macis, S.; Cibin, G.; Maggi, V.; Baccolo, G.; Hampai, D.; Delmonte, B.; D’Elia, A.; Marcelli, A. Microdrop Deposition Technique: Preparation and Characterization of Diluted Suspended Particulate Samples. Condens. Matter 2018, 3, 21. [CrossRef]

19. Dent, A.J.; Cibin, G.; Ramos, S.; Parry, S.A.; Gianolio, D.; Smith, A.D.; Scott, S.M.; Varandas, L.; Patel, S.; Pearson, M.R.; et al. Performance of B18, the Core EXAFS Bending Magnet beamline at Diamond. J. Phys. Conf. Ser. 2013, 430, 012023. [CrossRef]

20. Maggi, V.; Baccolo, G.; Cibin, G.; Delmonte, B.; Hampai, D.; Marcelli, A. XANES Iron Geochemistry in the Mineral Dust of the Talos Dome Ice Core (Antarctica) and the Southern Hemisphere Potential Source Areas. Condens. Matter 2018, 3, 45. [CrossRef]

C 2019 by the authors. Licensee MDPI, Basel, Switzerland. This article is an open access article distributed under the terms and conditions of the Creative Commons Attribution (CC BY) license (http://creativecommons.org/licenses/by/4.0/). 